\title{
A POMERON MODEL WHICH NEEDS NO DECOUPLING*
}

\author{
F. HENYEY and W.J. ZAKRZEWSKI** \\ Physics Department, University of Michigan, Ann Arbor, Michigan 48104, USA
}

Received 25 October 1972

\begin{abstract}
We present a model in which the Pomeron is a related pair of factorizable Regge poles both with intercept one. Our model requires a cut (fixed or moving) with a branch point intercept one. In this model the triple Pomeron vertex need not vanish and as a result the Pomeron may couple to asymptotically constant cross sections. Our model can even fit pp elastic scattering data moderately well.
\end{abstract}

A great deal of recent interest has focused on a series of theorems $[1-4]$ on the decoupling of the Pomeron, culminating in the following theorem [3]: If the Pomeron is a factorizable Regge pole of intercept one then it does not contribute to total cross sections. The entire series of theorems is based on the result of Abarbanel et al. [2] that, under the conditions specified above, the triple Pomeron vertex $\left(\Gamma_{p p p}\right)$ vanishes if all Pomerons have zero momentum transfer squared.

A key assumption in the proof ${ }^{\ddagger}$ of the vanishing of $\Gamma_{\mathrm{ppp}}$ is the assumption that the slope of the Pomeron trajectory is finite al $t-0$. In this paper we examine this assumption, and find a reasonable model with infinite slope trajectories in which this theorem cannot be established.

If the slope is to be infinite at $t=0$, the trajectory $\alpha(t)$ must be singular there ${ }^{\neq}$. Since the Froissart bound requires $\alpha(0) \leqslant 1$ (by assumption, $\alpha(0)=1$ for the Pomeron) a branch point is the only possible singularity. Thus, either at $t>0$ or at $t<0, \alpha(t)$ must be complex. Real analyticity then requires the existence of a complex conjugate trajectory in addition to the original one (i.e., $\alpha_{2}(t)=\alpha_{1}^{*}(t)$ ). Thus at least two trajectories are required to invalidate the theorem. In what follows we shall assume that the trajectories are complex for $t<0$. (Morc precisely there exists a

* Research supported by the U.S. Atomic Energy Commission.

** Present address: DAMTP, Silver Street, Cambridge, England.

$\neq$ We follow the proof of ref. [4].

$\neq \neq$ In any case, if the Pomeron has intercept one, it is singular at $t=0$. This is because it mixes with the multi-Pomeron cuts, all of whose branch points have intercept one. Later in the paper we investigate the question of whether this mixing suffices to give the desired result. range $-\left|t_{0}\right|<t<0$ in which the trajectories are complex.) We will refer to the two (or more) trajectories as different branches of the Pomeron.

The simplest possibility one might consider of this form is

$\alpha(t)=1+a_{1} \sqrt{t}+a_{2} t+a_{2} t^{3 / 2}+\ldots$

We shall establish that this trajectory is not satisfactory : the theorem still applies and this Pomeron must decouple.

In order to show this, and to discuss a model in which the theorem fails, we review the proof ${ }^{\ddagger}$ of the vanishing of the triple Pomeron vertex. The total cross section $a+b \rightarrow$ any thing cannot exceed any restricted part of itself. In particular, it cannot exceed the contribution of the "inclusive" cross section $a+b \rightarrow c+$ (any thing else with a large mass). In the limit that the center of mass energy is large compared to the large mass $M$ of the "anything else" (i.e., $s \gg M^{2} \gg m_{a}^{2}$ or $m_{\mathrm{b}}^{2}$ or $m_{\mathrm{c}}^{2}$ ) this contribution is dominated by the triple Pomeron diagram of fig. 1. The total cross sec-

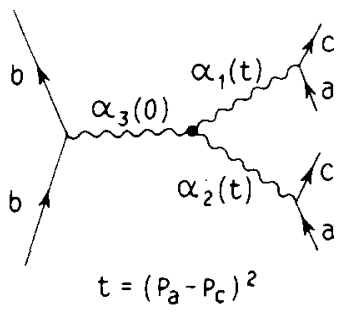

Fig. 1. The triple Pomeron diagram for the inclusive cross section $\mathbf{a}+\mathrm{b} \rightarrow \mathrm{c}+$ any thing. This diagram is dominant in a region $s=\left(P_{\mathrm{a}}+P_{\mathrm{b}}\right)^{2} \gg M^{2}\left(P_{\mathrm{a}}+P_{\mathrm{b}}-P_{\mathrm{c}}\right)^{2} \gg m_{\mathrm{a}}^{2}, m_{\mathrm{b}}^{2}, m_{\mathrm{c}}^{2}$, and $|t|<\epsilon_{t}$. Its total contribution in this region cannot exceed the total cross section. 
tion, a constant, must be greater than the contribution of fig. 1, which turns out to be approximately

const. $\int_{-\epsilon_{t}}^{0} \mathrm{~d} t \int_{1-\epsilon_{x}}^{1} \mathrm{~d} x\left(\frac{1}{1-x}\right)^{\alpha_{1}(t)+\alpha_{2}(t)-\alpha_{3}(0)}$

In this expression $x$ is the Feynman variable, approximately equal to $1-M^{2} / s ; \epsilon_{t}$ and $\epsilon_{x}$ are small fixed numbers chosen so that the triple Pomeron limit is valid to some desired degree of accuracy. The corrections to the approximations made in writing the above expression are irrelevant to our discussion. The constant which appears in front is proportional to the triple Pomeron vertex at $t=0$. This vertex is denoted by $\Gamma_{p}(0,0,0)$. The question of importance is whether $\Gamma_{p}(0,0,0)$ must be zero or whether it is allowed to be nonzero.

The answer depends on the convergence of the integral since we have constant $\geqslant$ const $\cdot$ integral. If the integral is convergent, $\Gamma_{\mathrm{p}}(0,0,0)$ can be chosen nonzero but small enough to satisfy the inequality. If, on the other hand, the integral is divergent, then $\Gamma_{p}(0,0,0)$ must be zero and the result of Abarbanel et al. is established.

The $x$ integration can be trivially done to give

integral $=\int_{-\epsilon_{t}}^{0} \mathrm{~d} t \frac{\epsilon_{x}^{\alpha_{3}(0)+1-\alpha_{1}(t)-\alpha_{2}(t)}}{\alpha_{3}(0)+1-\alpha_{1}(t)-\alpha_{2}(t)}$.

We are interested in the case $\alpha_{3}(0)=1$, and $\alpha_{1}(t)$ and $\alpha_{2}(t)$ may be the same of different branches of the Pomeron trajectory. Since $t$ is small $\alpha_{1} \approx \alpha_{2} \approx 1$, and $\epsilon_{x}^{2-\alpha_{1}-\alpha_{2}}$ may be replaced by $\epsilon_{x}^{0}=1$. Thus we have

either $\int_{-\epsilon_{t}}^{0} \frac{\mathrm{d} t}{2-\alpha_{1}(t)-\alpha_{2}(t)}<\infty$ or $\Gamma_{\mathrm{p}}(0,0,0)=0$.

The integral must be convergent for all pairs of trajectories $\alpha_{1}, \alpha_{2}$. (More precisely, we should sum the integrand over all possibilities.)

Let us examine this result for various cases. First is the simple case $\alpha_{1}(t)=\alpha_{2}(t) \approx 1+\alpha^{\prime} t$. Then

$\int_{-\epsilon_{t}}^{0} \overline{2}-\left.\alpha_{1} \frac{\mathrm{d} t}{(t)-\alpha_{2}(t)} \approx \frac{\ln |t|}{2 \alpha^{\prime} \mid}\right|_{t=-\epsilon_{t}} ^{0}=\infty$.

Therefore, the triple Pomeron vertex vanishes in this case. One can easily see that a finite set of finite slope poles all with intercept one cannot result in a nonzero triple Pomeron vertex. Second, let us try the square root trajectories

$\alpha_{1 \text { or } 2}(t)=1 \pm a_{1} \sqrt{t}+a_{2} t+\ldots$

If $\alpha_{1}$ and $\alpha_{2}$ are the same branch we have

$$
\left.\int_{-\epsilon_{t}}^{0} \frac{\mathrm{d} t}{2-\alpha_{1}(t)-\alpha_{2}(t)} \approx \frac{ \pm \sqrt{t}}{a_{1}}\right|_{-\epsilon_{t}} ^{0}<\infty
$$

However, if $\alpha_{1}$ and $\alpha_{2}$ are on different branches we have

$$
\begin{aligned}
& \int_{-\epsilon_{t}}^{0} \frac{\mathrm{d} t}{2-\alpha_{1}(t)-\alpha_{2}(t)} \approx \\
& \approx \int_{-\epsilon_{t}}^{0} \frac{\mathrm{d} t}{2-\left(1+a_{1} \sqrt{t}+a_{2} t\right)-\left(1-a_{1} \sqrt{t}+a_{2} t\right)} \\
& \quad=\left.\frac{1}{2 a_{2}} \ln |t|\right|_{-\epsilon_{t}} ^{0}=\infty
\end{aligned}
$$

since the positive and negative square roots cancel in the denominator. Therefore, even in the case of square root trajectories $\Gamma_{p}(0,0,0)=0$.

It is now clear what is required in order to have $\Gamma_{p}(0,0,0) \neq 0$. We assume eaxactly two branches, which must be complex conjugates of each other. We must have both

$$
\int \frac{\mathrm{d} t}{2-2 \alpha(t)}<\infty \text { and } \int \frac{\mathrm{d} t}{2-2 \operatorname{Re} \alpha(t)}<\infty .
$$

The second condition implies the first, so it is a necessary and sufficient condition to allow $\Gamma_{\mathrm{p}}(0,0,0) \neq 0$.

We have established that $\alpha(t)$ must have a branch point at $t=0$, and that a square root branch point is not sufficient. The real part of $\alpha$ must also be singular. In order not to violate the requirement that there not exist trajectories with $\operatorname{Re} \alpha>1$ for $t \leqslant 0$, there must be a $j$ plane cut with a branch point at $j=1$ when $t=0$, onto whose second sheet some branches of the Pomeron pole can move. (If the reader finds this last comment confusing he is advised to continue on to the following model where this effect will be discussed.) 
We are thus led to a "minimal" model which can allow the triple Pomeron coupling. The Pomeron trajectory in our model (at least near $t=0$ ) is given by

$\alpha(t)=1 \pm \mathrm{a} \sqrt{t} / \ln \left(t / t_{0}\right)$.

An appropriate branch of the logarithm is taken so that

$\operatorname{Re} \alpha(t)<1$ for $t<0$.

A correct branch, which we shall choose, is given by

$\alpha(t)=1+\frac{\alpha \sqrt{-t}\left[-\pi \pm i \ln \left(-t / t_{0}\right)\right]}{\pi^{2}+\ln ^{2}\left(-t / t_{0}\right)}$

and $a$ must be positive.

Of course, the logarithm has an infinite number of branches. How can we be justified in selecting only two of these branches and no others? The only possibility consistent with analy ticity is that all the other branches lie on unphysical sheets of some cut. This cut may have a moving branch point with intercept one, or the branch point may be fixed at $j=1$.

In fact, if the Pomeron has intercept one, the cuts caused by the exchange of two or more Pomeron poles all have intercept one. It is interesting to investigate whe ther these cuts can be those responsible for restoring analy ticity. Bronzan and Hui [8] have investigated the two Pomeron cut for an approximately square root trajectory. They find that a "quasistability" constraint requires that the pole-cut mixing occurs in the $t^{3 / 2}$ term of $\alpha(t)$ rather than in the $t^{1 / 2}$ term as we require. Their result can be modified of we allow the two poles to coalesce into a double pole at $t=0$, in which case the mixing is in the $t^{1 / 2}$ term. However, the observed constancy of the total cross section argues against this latter possibility, so we will not discuss it further. Thus it appears that the required cut is different from the two Pomeron exchange cut.

Another supporting bit of evidence is, as we will see, that the cut apparently interferes constructively with the pole, rather than distructively. Perhaps the cut we require is caused by the type of mechanism proposed by Auerbach et al. [5].

We can exhibit an amplitude which explicitly includes such a cut, and which is analy tic at $t=0$. We want this amplitude to essentially reproduce the Regge pole trajectory $\alpha(t)=1 \pm a \sqrt{t} \ln \left(t / t_{0}\right)$. Near $j=\alpha(t)$ we have $\ln (j-1)=\ln a+\frac{1}{2} \ln t+\ln \ln \left(t / t_{0}\right) \approx \frac{1}{2} \ln t$ near $t=0$. Therefore $\left[j-1 \pm \frac{1}{2} a \sqrt{t} \ln \left\{(j-1) / j_{0}\right\}\right]^{-1}$ has a pole near $j=\alpha(t)$. The sum of these two factors yields an amplitude $B(t)(j-1) /\left[(j-1)^{2}-\frac{1}{4} a^{2} t \ln ^{2}\left\{(j-1) / j_{0}\right\}\right]$ This amplitude is explicitly analytic at $t=0$, and it has a fixed cut at $j=1$ from the logarithm. At positive $t$ it has one pole on the physical sheet of that cut and two poles very close to the physical sheet on the second sheet. At negative $t$ it has a pair of complex conjugate poles and a rather weak cut. Moreover it has the property that

$\lim _{t \rightarrow 0^{-}}\left(\frac{\mathrm{d}}{\mathrm{d} t} \operatorname{Re} \alpha(t)\right)=\infty$

and it allows a finite $\Gamma_{\mathrm{ppp}}(0)$. This amplitude is an explicit counterexample to the decoupling theorems.

Can such a Pomeron as we have described possibly agree with experimental data for elastic reactions at very high energy? We have attempted to fir the pp elastic data [6] obtained at the CERN intersecting storage rings. We parameterize the residue function as an exponential in $t$, so that our amplitude becomes

$A \mathrm{e}^{B t}\left[\left(\frac{s}{s_{0}}\right)^{\alpha_{+}(t)}+\left(\frac{s}{s_{0}}\right)^{\alpha_{-}(t)}\right]$

with $\alpha$ given by the appropriate branches of

$\alpha_{ \pm}(t)=1 \pm a \sqrt{t} / \ln \left(t / t_{0}\right)$

as discussed above. We neglect the contribution from the cut which we know must be present, since we know very little about its discontinuity.

The fit is shown in fig. 2. We use the followubg parameters: $A=50.9, B=3.5 \mathrm{GeV}^{-2}, a=0.645 \mathrm{GeV}^{-1}$, $s_{0}=t_{0}=1 \mathrm{GeV}^{2}$. Our fit is good for $|t|<0.1$, but cannot be considered adequate beyond that point. Our Pomeron shows too much shrinkage there. We do show an improvement however, over a fit with a single linear trajectory whose slope is given by the shrinkage for $|t|<0.1$. If the basic idea of our model is correct, the discrepancy with the data may be caused by several features. It may be that the real parts of our trajectories fall too fast at larger $|t|$, where they are not constrained by the necessary behavior near $t=0$. Or, more likely, it may be that the neglected cut begins to play a role at $t \approx-0.1$. In this case, the cut must interfere constructively with the poles in order to decrease the rate of shrinkage.

Another possible difficulty with our fit is that, even if our basic idea is correct, our detailed model is wrong. 


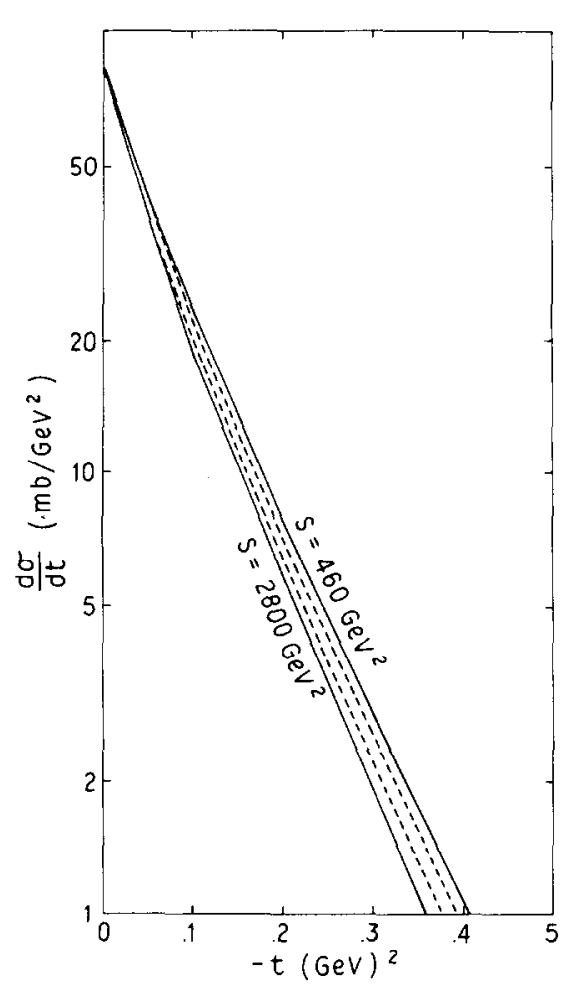

Fig. 2. The fit to elastic pp scattering. The solid lines are the theoretical curves at $s=460$ and 2800 , and the dot ted curves are the experimental values. The excessive shrikhage at $|t|>0.1$ may be due to neglected curvature of the trajectory or to the neglected cut.

One may find many trajectories whose real part has an infinite slope which allows the theorem to be violated. For example, $\alpha(t)=1+a t \ln ^{2} t$ suffices. It is not our purpose here to distinguish between such models, but only to point out that such models exist and can de- cribe the data better than Regge poles of the canonical from $\alpha(t)=1+\alpha^{\prime} t$.

It would be possible for our mechanism to allow the Pomeron to contribute to total cross sections and yet not be phenomenologically visible. This can happen because the required effect is extremely small. Chew [7] has estimated that if the Pomeron pole has a straight line trajectory, its intercept has only to be lowered to 0.998 to satisfy the inequality on which the theorem is based. Likewise, our mechanism only requires a very small coefficent of the singular term in the trajectory.

In summary it is still possible that the Pomeron is primarily a pair of factorizing Regge poles with intercept one. These poles become a single factorizing pole at $t=0$. Such a model is not wildly inconsistent with phenomenological considerations and is superior from theoretical considerations to all simpler pole models.

We would like to thank G.L. Kane for help with the data fitting and providing us with his computer programs, J.J.G. Scanio for suggesting the precise form of the trajectory used in our model, and F. Low for a stimulating conversation.

\section{References}

[1] J. Finkelstein and K. Kajantie, Nuovo Cim. 56 A (1968) 659.

[2] H. Abarbanel et al., Phys. Rev. Lett. 26 (1971) 937.

[3] R. Brower and J. Weis, MIT preprint (1972) 280.

[4] C.E. Jones et al., Phys. Rev. D6 (1972) 1033.

[5] S. Auerbach et al., Phys. Rev. Lett. 29 (1972) 522.

[6] G. Barbiellini et al., Phys. Lett. 39B (1972) 663.

[7] G. Chew, XVI Inter. Conf. on High energy physics.

[8] J.B. Bronzan and C.S. Hui, Phys. Rev. D5 (1972) 964. 